Investigaciones Fenomenológicas, n. 14, 2017, 13-29.

e-ISSN: $1885-1088$

\title{
MERLEAU-PONTY Y LA PSICOLOGÍA DE LA FORMA*
}

\section{MERLEAU-PONTY AND THE GeSTALT PSYCHOLOGY}

\author{
Renaud Barbaras \\ Universidad Paris 1 \\ Traducción: Manfredi Moreno \\ paramanfredi@gmail.com
}

\begin{abstract}
Resumen: A partir del análisis de la relación de Merleau-Ponty con la psicología de la forma, se intenta dar una lectura de su fenomenología como una lectura ontológica de la forma. De este modo, Merleau-Ponty no solo puede pensar una alternativa a la fenomenología de Husserl, sino que le permite elaborar su propia idea de Ser y dar una nueva interpretación de la diferencia ontológica de Heidegger. Desde una descripción fenomenológica de la experiencia perceptiva, a partir de las nociones de comportamiento, forma y estructura, se mostrará enseguida el sentido de ser que moviliza una filosofía de la forma que implicará proyectar un pensamiento de una pertenencia radical de la fenomenalidad a la existencia, y además renovar el sentido de una fenomenología genética que considera toda conciencia trascendental en el marco de una facticidad originaria.
\end{abstract}

Palabras clave: forma, comportamiento, estructura, percepción, fenomenología

\begin{abstract}
On the basis of the relationship Merleau-Ponty establishes with Gestalt psychology, I seek to provide an interpretation his phenomenology as an ontological reading of the notion of form. Therefore, I claim that Merleau-Ponty is able, not only to develop an alternative to Husserl's phenomenology, but also to elaborate his own idea of Being; in addition, he can offer a new understanding of the Heidegger's ontological difference. From a phenomenological description of perceptive experience, and starting with the notions of behavior, form and structure, I will then show the sense of being mobilized by a philosophy of form. This sense of being implies a projection of a thinking of phenomenality's radical belonging to the existence and, also, renewal of a genetic phenomenology that considers the whole of transcendental consciousness within the framework of an originary facticity
\end{abstract}

Keywords : form, behavior, structure, perception, phenomenology

"Barbaras, R. (2001/2). "Merleau-Ponty et la psychologie de la forme", Les Etudes philosophiques, Paris, PUF, p. 151-163. Publicado con autorización, para esta revista, por parte de Les Études philosophiques, por lo que los editores de Investigaciones Fenomenológicas les agradecen sinceramente. 
A pesar de su aparente modestia, la cuestión de la relación de Merleau-Ponty a la psicología de la forma no podría ser tratada satisfactoriamente sino en el marco de un libro. En efecto, no se exagera al decir que, desde el principio hasta el final, Merleau-Ponty intentó pensar la forma, descubierta por la Gestaltpsychologie, y que, en ese sentido, la forma hace las veces de la "cosa misma" a la cual el precepto husserliano nos ordena retornar: todas las descripciones merleaupontianas, desde la del comportamiento hasta la del mundo percibido, son guiadas por la Gestalt y como animadas por esta. Desde este punto de vista, habría que hacer una distinción entre la cuestión de la psicología de la forma, frente a la cual Merleau-Ponty toma tempranamente una posición definitiva, y la cuestión de la forma, que lo ocupa a lo largo de su obra. Asimismo, como se da a entender desde La estructura del comportamiento ${ }^{1}$, su proyecto es construir una filosofía de la forma que prolonga la psicología de la forma, es decir, supera sus limitaciones filosóficas. Todo indica que el concepto de forma, que resulta de una descripción fiel de los fenómenos, deshace las categorías espontáneas de la actitud natural, e incluso de la tradición filosófica, siendo la razón por la cual la cuestión de la significación y del estatuto ontológico de la forma se encuentra en el centro de su pensamiento.

Ahora bien, se pueden discernir al menos dos momentos. La referencia a la Gestaltpsychologie es omnipresente en La estructura del comportamiento y en las dos primeras partes de la Fenomenología de la percepción. Sin embargo, en los años que siguen a la Fenomenología de la percepción, Merleau-Ponty se aleja manifiestamente de la psicología de la forma. (Él) se orienta hacia la lingüística y hacia una reflexión sobre el arte, lo que es perfectamente coherente puesto que, al término de la Fenomenología de la percepción, la cuestión que queda pendiente a su parecer es aquella del estatuto de la idealidad y, en términos más generales, de la razón. Ahora bien, la Gestalt vuelve a aparecer en las notas de trabajo de Lo visible y lo invisible, siendo abordada en otros términos: ya no es invocada como muestra descriptiva para criticar el pensamiento objetivo en sus dos formas, intelectualismo y empirismo - como fue el caso hasta la Fenomenología de la percepción- sino que es pensada por ella misma y positivamente

${ }^{1}$ Merleau-Ponty, M. (1942). La structure du comportement, París: PUF, p. 143 (en adelante abreviada $\mathrm{SC})$. Nota del traductor: cada referencia alude a la paginación francesa con traducción de mi autoría (siempre consultando la traducción en español existente). 
como aquello que "tiene la clave para el problema del espíritu"2 y que nos obliga a una revisión profunda de nuestras categorías. La filosofía de la forma, por la cual Merleau-Ponty aboga, parece entonces confundirse con una ontología. Asimismo, mientras que, inicialmente, la forma se utiliza en favor de una filosofía de la conciencia, contra la interpretación naturalista de la psicología, ella viene a constituir el instrumento mismo de la crítica de la filosofía de la conciencia y de las categorías que la sostiene. Querría preguntarme aquí sobre esta inversión y en consecuencia sobre esta doble presencia de la Gestalt, para tratar de mostrar que si la Gestaltpsychologie le ofrece en primer lugar a Merleau-Ponty un concepto de cogito que representa una alternativa con relación a Husserl, la noción de forma, pensada por ella misma, le permite elaborar una idea del Ser y de la diferencia ontológica que representan quizás una alternativa respecto a Heidegger.

Lo que está en juego en La estructura del comportamiento es la redefinición de la relación entre la conciencia y la naturaleza y es por esto por lo que MerleauPonty toma como objeto una noción neutra frente a la distinción de lo psíquico y de lo orgánico, la del comportamiento. Pero, en vez de proceder a un análisis fenomenológico del comportamiento en tanto que vivido, se apoya en los resultados del conocimiento objetivo, es decir, precisamente en los de la psicología de la forma y de la fisiología, de los cuales procede. Como lo escribe en la Fenomenología de la percepción: "Sin querer prejuzgar nada, tomaremos al pie de la letra el pensamiento objetivo y no le haremos preguntas que él no se haga a sí mismo. Si nos vemos conducidos a encontrar la experiencia detrás de él, este

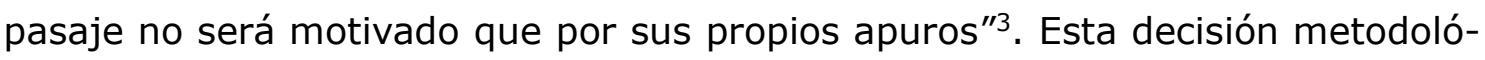
gica es capital: representa en cierta medida la versión merleau-pontiana de la reducción fenomenológica. En efecto, mientras que un análisis reflexivo del comportamiento habría conducido casi inevitablemente a la posición de una conciencia transparente ("el cogito no nos enseña de una vez por todas que no tendría-

\footnotetext{
2 Merleau-Ponty, M. (1964). Le visible et l'invisible, Paris: Gallimard, p. 246 (en adelante abreviada VI). $\mathrm{PhP})$.

${ }^{3}$ Merleau-Ponty, M. (1945). Phénoménologie de la perception, Paris: Gallimard, p. 86 (abreviada
} 
mos el conocimiento de nada si no tuviésemos primero el de nuestro pensamiento") 4 , el descubrimiento de la forma nos conduce ciertamente a la conciencia, pese a los prejuicios naturalistas de la psicología de la forma, pero a una conciencia perceptiva que no puede ser más asimilada al ego trascendental kantiano o husserliano, y que queda por definir. Así, el análisis del estatuto de la forma abre el campo de la percepción, que es comprendido originariamente en el marco de la observación científica del comportamiento, como percepción de una forma orgánica por un espectador extranjero, es decir, como un modo de la intersubjetividad.

Merleau-Ponty se opone principalmente a la reflexología de Pavlov, que consiste en reconstruir el comportamiento de manera longitudinal como una reacción característica a un estímulo objetivo específico, según una vía nerviosa definida. Entonces él hace suya la distinción establecida por Koffka entre "el ambiente geográfico", que corresponde a la realidad física en la que el individuo se mueve y "el ambiente del comportamiento" que define aquello ante lo cual el animal reacciona, es decir, su propio medio. Así, aquello a lo que el animal es sensible no corresponde a un objeto del mundo geográfico (que, cabe señalar, no es otra cosa que el ambiente del comportamiento del hombre en la actitud de conocimiento) sino a unas estructuras, de las que los elementos del ambiente geográfico son solo el motivo y que él constituye por así decirlo él mismo, en tanto que ellas son correlativas de sus a priori biológicos. Por eso, con respecto al comportamiento reflejo, tal animal no reaccionará a colores absolutos sino a una relación, es decir, a un tipo de contraste que puede transponerse en varios tonos. Estas conclusiones se confirman en los comportamientos superiores, donde la contribución de Goldstein resulta decisiva. En efecto, el estudio de lesiones cerebrales permite desechar la idea de una correspondencia biunívoca entre localizaciones cerebrales y sectores del comportamiento. En realidad, muchas lesiones locales tienen una incidencia estructural, es decir, afectan a un nivel del comportamiento (por ejemplo, la aptitud simbólica) más que a un contenido. Como señala Merleau-Ponty, "una lesión, aun localizada, puede determinar trastornos de estructura que abarcan el conjunto del comportamiento, y trastornos de estruc-

${ }^{4}$ SC, p. 138. 
tura similares pueden ser provocados por lesiones situadas en diferentes regiones de la corteza"5. Por supuesto, no se debe concluir que volvemos a caer en una forma de vitalismo porque, si cada parte del cerebro expresa un comportamiento total, no deja de ser cierto que la localización de la lesión no es indiferente: "No se puede tratar el funcionamiento nervioso como un proceso global donde todas las partes del sistema intervendrían de la misma manera. La función no es jamás indiferente del substrato por el que se realiza"6: en otros términos, la ubicación de las lesiones determina el punto de aplicación principal de los trastornos de estructura. Por consiguiente, es preciso concebir el funcionamiento cerebral como un proceso del tipo figura-fondo: un comportamiento circunscrito es inseparable de un fondo que él constituye como un modo específico de expresión. Por eso Merleau-Ponty muestra que, sea cual sea el plano en el que es examinado, el comportamiento puede ser descrito mediante el concepto de forma, tal como Koehler lo define, a saber, como

procesos totales cuyas propiedades no son la suma de aquéllas que poseerían las partes por separado, [...] como procesos totales que pueden ser indistinguibles el uno del otro mientras que sus "partes", comparadas cada una entre sí, difieren en tamaño absoluto, o sea como todos transponibles ${ }^{7}$.

Merleau-Ponty no se queda ahí: se pregunta sobre el estatuto de la forma, sobre su sentido de ser. En efecto, si bien la Gestaltpsychologie se opone a un modo de pensar analítico, no considera que "el atomismo psicológico sólo es un caso particular de un prejuicio más general: el prejuicio del ser determinado"8, de manera que su propósito sería el de inscribir la Gestalt en el marco de una naturaleza existente en sí. Tal es el sentido de la teoría del isomorfismo de Koffka. Aduciendo que de hecho encontramos formas en el universo físico (distribución de cargas eléctricas en un conductor elipsoide), este "plantea las formas vividas como réplicas interiores de formas externas del mundo físico, valiéndose de formas del sistema nervioso"9. Así, la organización fenomenal del ambiente del comportamiento hace referencia en última instancia, por isomorfismo, a una

5 SC, p. 66.

${ }^{6}$ SC, p. 76.

7 SC, p. 49.

$8 \mathrm{PhP}$, p. 62. 441.

9 Merleau-Ponty, M. (1988). Merleau-Ponty à la Sorbonne. Résumés de cours 1949-1952, Cynara, p. 
estructura física. Ahora bien, todo el esfuerzo de Merleau-Ponty va a consistir en mostrar que existe una contradicción entre la significación misma de la forma y la tentativa de inscribirla en una ontología de tipo naturalista. Aparte los numerosos problemas que supone (en particular el de la posibilidad de una psicología social), la idea de una forma física no puede tener sentido:

Considerada como un ser de naturaleza, existiendo en el espacio, la forma siempre se dispersaría en varios lugares, se distribuiría en eventos locales, aunque esos hechos se entredeterminen; decir que ella no sufre esta división equivale a decir que no está desplegada en el espacio, que no existe como cosa, que es la idea en la que se reúne y se reduce lo que sucede en varios lugares. Esta unidad es la de los objetos percibidos. ${ }^{10}$

En otras palabras, un Todo que no se confunda con la suma de sus partes no posee sino una existencia fenomenal y pensar la realidad del comportamiento o del organismo como forma es plantear la identidad de su realidad y su fenomenalidad. En ello, no es sino la psicología de la forma misma quien se ve obligada a suspender la ontología ingenua de la que había partido, siendo así conducida por su cuenta hacia la actitud fenomenológica: Merleau-Ponty se interesa aquí por la ciencia porque la ciencia se hace fenomenología.

El análisis que propone de las estructuras vitales, procedente en su conjunto de Goldstein, así lo confirma. El ser vivo no puede nunca remitirse al nivel de procesos físico-químicos; para hacer aparecer un organismo, se necesitan trazar líneas de separación, elegir puntos de vista de donde ciertos conjuntos reciben una significación común y aparecen como fenómenos de nutrición por ejemplo, del mismo modo como un conjunto de movimientos objetivos se presentará como un gesto de fuga. La vida como tal sólo es accesible en el plano de totalidades irreductibles al conjunto de sus partes. Pero este rechazo del mecanicismo no equivale a un vitalismo puesto que, si el todo se distingue de la suma de sus partes, este no es otra cosa que ellas y no puede entonces remitir a fuerza vital alguna: la totalidad se distingue de las partes en tanto que ella constituye su significación para una conciencia, sin que sea así una substancia distinta a la substancia material. La idea de significación permite por tanto dar cuenta de la

${ }^{10}$ SC, p. 156. 
especificidad de la vida sin introducir una fuerza vital: la originalidad de la vida remite en última instancia a la del plano fenomenal. Por eso Merleau-Ponty puede escribir:

La significación y el valor de los procesos vitales, que la ciencia se ve obligada a considerar, son los atributos del organismo percibido, pero no son sin embargo unas denominaciones extrínsecas con respecto al organismo verdadero, porque el organismo verdadero, aquél que la ciencia considera, es la totalidad concreta del organismo percibido, portador de todas las correlaciones que en él descubre el análisis sin ser descomponibles en ellas ${ }^{11}$.

Si el alcance crítico de este análisis es inequívoco - la psicología de la forma permite suspender la ontología ingenua en favor de unos seres que remiten por esencia a una conciencia-, su significación positiva lo es menos. ¿Cuál es el sentido de ser de la forma en tanto que irreductible a un ser natural? ¿En qué consiste la percepción en tanto que alcanza la significación junto a la existencia o la existencia como significación? Después de todo, se trata de pensar la identidad del ser y de la fenomenalidad de modo que no se comprometa su diferencia, que es exigida por la idea misma de fenomenalización: una cosa parece y se distingue pues de alguna manera de su aparecer. Los extensos análisis que Merleau-Ponty dedica a esta cuestión en la última parte de La estructura del comportamiento son claramente embarazosos. Este, sin duda, porque su principal adversario es el naturalismo, hace hincapié sobre el significado crítico, en sentido kantiano, de sus conclusiones. El organismo, escribe Merleau-Ponty, no es el producto real de una naturaleza exterior sino "una unidad de significación, un fenómeno en sentido kantiano"12 y reconoce que todos sus análisis conducen "a la actitud trascendental, es decir a una filosofía que aborda toda realidad concebible como un objeto de conciencia"13. No obstante, no puede obviamente asumir hasta el final el idealismo trascendental, ya que eso equivaldría a negar lo que distingue la Gestalt de una simple significación y que constituye toda la contri-

\footnotetext{
${ }^{11}$ SC, p. 169.

12 SC, p. 172.

13 SC, p. 217.
} 
bución de la psicología de la forma. Por esa razón también afirma que sus conclusiones se encuentran "en una relación de simple homonimia" con la filosofía criticista:

lo que hay de profundo en la Gestalt de donde hemos partido, no es la idea de significación, sino aquella de estructura, el cruce de una idea y de una existencia indiscernibles, el acuerdo contingente por el cual los materiales empiezan a tener sentido ante nosotros, la inteligibilidad en estado naciente ${ }^{14}$.

El problema de la percepción, al cual la forma nos introduce, se plantea aquí en toda su agudeza. En tanto que la forma es un modo de unidad irreductible a sus partes, hay que admitir que ella es constituida por una conciencia, pero en tanto que esta unidad no es la de una significación ideal sino de una estructura inmanente a las partes, hay que incorporar a esta conciencia la dimensión misma de la existencia, es decir finalmente la de su otro. La forma plantea el problema, al cual Merleau-Ponty no dejará más de enfrentarse, de una fenomenalidad indiscernible de una existencia bruta, de un sentido retenido en el espesor de una materia y, por ende, de una conciencia que es capaz de pasividad encontrándose por ello inscrita en aquello mismo que ella hace aparecer; esta es la razón por la que el problema de la percepción, planteado a partir de la forma, se confunde con el problema de la encarnación.

Puesto que la unidad entre los términos puestos en juego - todo-partes, forma-materia, significado-existencia, conciencia-cuerpo - no pueden pensarse de manera inmediata, la solución esbozada por Merleau-Ponty será inevitablemente dialéctica. En efecto, mientras que el pensamiento criticista rechaza indefinidamente el problema de la pasividad y de la encarnación, puesto que, observa Merleau-Ponty, no hay cuerpo que no sea objeto para una conciencia,

es a cada momento para nosotros que la conciencia experimenta su inherencia a un organismo, puesto que no se trata de una inherencia a unos aparatos materiales, los cuales no pueden ser en realidad solo objetos para la conciencia, sino una presencia a la conciencia de su propia historia y de las etapas dialécticas que ella ha atravesado

${ }^{14}$ SC, p. 223. 
[...]. El espíritu no utiliza el cuerpo sino que se hace a través de él al mismo tiempo que lo transfiere fuera del espacio físico ${ }^{15}$.

Finalmente, la unidad de la existencia y de la esencia en la forma no puede pensarse sino sobre el modo dialéctico de una identidad mediata, donde cada término es la negación del otro: la existencia es la significación aún fuera de sí y la significación la existencia devenida aquello que ella es, es decir para sí. Correlativamente, por parte del sujeto, pasividad y actividad, encarnación y conciencia no son sino los momentos abstractos de un proceso por el cual la conciencia tiene acceso a ella misma: el cuerpo, es la conciencia entendida del punto de vista de su génesis y la conciencia el cuerpo entendido del punto de vista de su verdad. Desde luego, tal perspectiva deja un sentimiento de disconformidad en la medida en que renuncia al respeto fenomenológico de lo que se encuentra estrictamente implicado en la experiencia de la forma a favor de un punto de vista especulativo y abstracto. En realidad, Merleau-Ponty se contenta con esbozarlo y este pasaje al límite debe ser comprendido como el índice de un problema más que como una verdadera solución. La conclusión de la obra muestra que lo cierto es que el problema del estatuto de la forma y, por tanto, el de la percepción siguen sin resolverse: las formas son objetos "que solo existen por su sentido, pero el sentido que emerge en ellos no es aún un objeto kantiano, la vida intencional que los constituye no es todavía una representación, la 'comprehensión' que permite acceder a ellos no es aún una intelección"16.

Es este problema que la Fenomenología de la percepción se propone abordar. Para este fin, Merleau-Ponty abandona el punto de vista externo del científico sobre el comportamiento a favor de una descripción fenomenológica de la experiencia perceptiva. La psicología de la forma es de nuevo movilizada, sin embargo, Merleau-Ponty se basa esta vez en la psicología de la percepción propiamente tal, es decir, en el estudio de las formas perceptivas. Es lo que le permite, a lo largo del libro, criticar simétricamente el empirismo y el intelectualismo. Efectivamente, en tanto que lo originario perceptivo es una forma, es preciso recusar el empirismo, que trata de componer la cosa a partir de sensaciones simples y confunde así el orden lógico con el orden trascendental. No obstante,

${ }^{15}$ SC, p. 225.

16 SC, p. 241. 
la psicología de la forma desempeña un papel fundamental en lo que viene de alguna manera a rectificar la inspiración intelectualista de la perspectiva husserliana, que Merleau-Ponty aprueba ahora firmemente. En efecto, Husserl tiende a someter la donación por esquicios [esquisses] -determinación eidética de lo percibido- a las exigencias de la fenomenología de la razón, que identifica existencia y posibilidad de una donación adecuada, con lo cual la retirada de la cosa en el esquicio es inevitablemente entendida como inadecuación. Merleau-Ponty, al contrario, se basa en la psicología de la forma para poner de manifiesto la irreductibilidad de la donación por esquicios, es decir, la imposibilidad de principio de una donación adecuada del objeto percibido, imposibilidad que se expresa precisamente en la relación necesaria de la figura al fondo. Con ello es conducido incluso a criticar la dependencia del cogito husserliano frente al cogito cartesiano y a reconsiderar la importancia de la fenomenología genética, que pone de manifiesto la relación constitutiva de la conciencia trascendental con una facticidad originaria.

Sin embargo, en cuanto a lo esencial, la Fenomenología de la percepción no representa un verdadero avance en relación con La estructura del comportamiento. La noción de forma permite ciertamente a Merleau-Ponty circunscribir negativamente las condiciones del problema de la percepción y distanciarse de este modo de la tradición. Pero, en la medida en que no cuestiona radicalmente las categorías fundamentales que Husserl hereda de esta misma tradición, no logra sacar provecho plenamente de las potencialidades filosóficas de la forma. De ello se desprende que la definición que da de la conciencia perceptiva procede por exclusión y no se distingue en nada de aquella con la que concluye La estructura del comportamiento: "La conciencia no es ni posición de sí, ni ignorancia de sí, es no disimulada a sí misma, es decir que nada hay en ella que no se anuncie de alguna forma a ella, aun cuando no tenga necesidad de conocerlo expresamente"17. Por último, como es sabido, Merleau-Ponty resuelve el problema de la percepción y de la encarnación recurriendo a la temporalidad originaria, tal como fue elaborada por Husserl. En tanto que cada presente se sobrepasa hacia el futuro, existe una síntesis de lo percibido, el aspecto sensible se da

${ }_{17}$ PhP, p. 342. 
como aspecto de algo; pero en tanto que dicho rebasamiento es él mismo temporal, que dicho futuro será el presente de un nuevo futuro, en resumen, en tanto que el tiempo no se totaliza, la experiencia perceptiva sigue siendo parcial y el objeto inevitablemente trascendente. Del mismo modo, la conciencia está encarnada en tanto que ella es temporal pero el cuerpo no es jamás un objeto y deja siempre transparecer un sentido ya que el presente es su propio rebasamiento hacia el futuro. Como lo escribe Merleau-Ponty a propósito del estatuto del cuerpo: "Lo único verdadero es que nuestra existencia abierta y personal descansa sobre una primera base de existencia adquirida y fija. Pero no sabría ser de otro modo si nosotros somos temporalidad, puesto que la dialéctica de lo adquirido y del futuro es constitutiva del tiempo"18. Se podría decir que este recurso al presente vivo husserliano es el equivalente exacto de la referencia a Hegel en La estructura del comportamiento. Merleau-Ponty entiende como temporalidad originaria lo que antes era concebido como dialéctica del en-sí y del para-sí; pero con ello no supera la perspectiva dialéctica, dándole solamente una figura efectiva, la de la temporalidad que, en Hegel mismo, es el ser-ahí del concepto. Así, al igual que en La estructura del comportamiento, la dialéctica viene a subsanar la diferencia entre lo que hay que pensar y la serie de dualidades a partir de las cuales intentamos pensarlas (en-sí, para-sí; existencia, esencia; materia, forma); en esto, (ella) encubre una dificultad más que la resuelve. Esta dificultad consiste en la inadecuación de las categorías aplicadas frente a la experiencia que ellas intentan dar cuenta, en concreto la de la forma. En resumen, durante todo este periodo, el estudio de la Gestalt allana el camino de la experiencia bruta y constituye de este modo la puesta en marcha de la crítica del empirismo y del intelectualismo, pero la Gestalt nunca es pensada por sí misma.

Es precisamente de esta dificultad que Merleau-Ponty toma conciencia en los años que siguen a la Fenomenología de la percepción y es lo que va motivar el pasaje hacia una perspectiva ontológica. Empieza a comprender poco a poco la imposibilidad de dar cuenta de lo percibido en el marco de una filosofía de la conciencia y, en consecuencia, de la irreductibilidad de la experiencia a la serie de conceptos por los que se intenta describirla: materia-forma, objeto-sujeto,

18 PhP, p. 494. 
etc. Desde este punto de vista, resulta sumamente significativo que los dos capítulos críticos más relevantes de Lo visible y lo invisible estén dedicados a la dialéctica, por una parte, y a la fenomenología, por otra, en tanto que ambas dependen de la dualidad del hecho y de la esencia, rasgo inconfundible del pensamiento objetivo. Por tanto, los prejuicios que Merleau-Ponty denuncia son también los suyos, por lo menos hasta Lo visible y lo invisible. Es de destacar asimismo que la temporalidad pierde la preponderancia que disfrutaba hasta ese momento. Finalmente, pensamiento dialéctico y pensamiento de la temporalidad originaria son avatares de la filosofía de la esencia: su función sigue siendo la de salvar la desgarradura originaria del fenómeno a partir del hecho y de la esencia, la existencia y la significación. Entonces se trata para Merleau-Ponty de volver a lo percibido mismo, antes de esta desgarradura, es decir, determinar el sentido de ser del Ser a partir de lo percibido, que es la forma originaria, en lugar de subordinarlo a una ontología implícita -en definitiva, pensar según lo percibido en lugar de pensar lo percibido según el hecho y la esencia. En otras palabras, se trata de pensar la percepción a partir de lo percibido, considerado como un sentido de ser originario, en lugar de dar cuenta de lo percibido a partir de la percepción entendida como el acto de una conciencia, puesto que el recurso a la conciencia nos conduce inevitablemente a la escisión del fenómeno. Se entiende así la importancia que Merleau-Ponty atribuye a la forma en el periodo final de su pensamiento: con la forma, la filosofía dispone de un concepto adecuado para la figura originaria de lo percibido y sólo hay que pensarla por sí misma para refundar nuestras categorías. La ontología de Merleau-Ponty procede de la consideración de la forma no sólo más allá de los prejuicios naturalistas de los psicólogos sinotambién más allá de los prejuicios dualistas del filósofo mismo. Es en este sentido que podemos comprender esta afirmación sorprendente, relativa a la Fenomenología de la percepción pero que puede ser ampliada a todo el primer periodo: "Debo mostrar que todo aquello que podríamos considerar como psicología es en realidad ontología"19.

Esta puesta en obra de la Gestalt para la elaboración de una ontología es asumida explícitamente por Merleau-Ponty. Es necesario mostrar, nos señala,

${ }^{19}$ VI, p. 230. 
"que estas nociones (pregnancia, Gestalt, fenómeno) representan una toma de contacto con el ser en tanto puro darse. Asistimos a este acontecimiento por el cual algo se da"20. Dicho de otra manera, la Gestalt no es la cosa sino el acontecimiento de su aparición o de su constitución, a saber, la cosa en estado naciente, anterior a toda forma de objetivación o de idealización, lo que él Ilama Etwas; la forma no es un apareciente entre otros sino la estructura misma del aparecer. Es en este sentido que puede afirmar: "Figura sobre fondo, el más simple Etwas -la Gestalt tiene la clave del problema del espíritu"21. Es por lo tanto coherente que Merleau-Ponty reconozca que "toda psicología que vuelva a situar la Gestalt en el marco del 'conocimiento' o de la 'conciencia' pierde el sentido de la Gestalt"22, recusando con ello la manera en que la abordaba él mismo en sus obras anteriores. Por consiguiente, ¿cómo definirla? Merleau-Ponty rechaza las definiciones negativas, que retomaba por su cuenta en La estructura del comportamiento y que, en realidad, remiten a una ontología objetivista: "¿Qué es una Gestalt? Un todo que no se reduce a la suma de las partes, definición negativa, exterior Descripción de la Gestalt por oposición al ámbito de en-sí en el que uno se instala"23. ¿Cómo caracterizarla positivamente? Faltan las palabras, puesto que nuestra lengua misma está estructurada con arreglo a las categorías de la ontología objetiva, y es por lo que Merleau-Ponty fluctúa entre varios registros metafóricos: "Es un principio de distribución, el pivote de un sistema de equivalencias, es el Etwas cuyos fenómenos parcelarios serían su manifestación"24. Es el sistema de equivalencias, afirma en substancia en otra parte, alrededor del cual cada cosa sensible se establece y del cual el trazo del pintor, la "línea sinuosa", es la evocación perentoria. Se trata entonces de una unidad sin concepto o sin principio, que no se distingue de la diversidad que ella unifica y que solo parece por tanto bajo la forma de una equivalencia no tematizable a los momentos que articula: es la unidad de un estilo que está presente sin ser nunca dado temáticamente, como un tema que solo aparece en sus variaciones. En suma, la

\footnotetext{
${ }^{20}$ VI, p. 259.

21 VI, p. 246.

22 VI, p. 259.

${ }^{23}$ VI, p. 258.

${ }^{24}$ VI, p. 258.
} 
forma es la identidad inmediata de lo uno y de lo múltiple. De este modo, en lugar de hablar de formas más o menos pregnantes como lo hace la psicología de la forma, habría que definir el modo de existir de la forma por medio de la pregnancia, en el sentido de aquello que es omnipresente sin ser asignable, de aquello que penetra los contenidos sin poder ser localizado o delimitado, de aquello que está ahí escapando al mismo tiempo a la extensión.

No cabe duda entonces que la forma desbarata las oposiciones tradicionales $y$, en primer término, la del hecho y de la esencia, al punto que es porque ha tomado conciencia de la necesidad de superar esta oposición que Merleau-Ponty pudo esbozar una verdadera filosofía de la forma ("mostrar en la Introducción que se necesita absolutamente superar la bifurcación esencia-existencia: Wesen (verbal). Demostrarlo a partir de la Gestalt"25). En efecto, como pregnancia, la forma no es del orden de la idea o de la esencia ya que conserva un lazo espaciotemporal, incluso si, por otra parte, existe en el modo de la proyección y no se encuentra entonces propiamente localizada; es una "significación cargada", desmoronada en el espacio mientras al mismo tiempo se distingue de la materia:

La Gestalt no es un individuo espacio-temporal, está preparada para integrarse a una constelación que atraviesa el espacio y el tiempo, - pero no es libre con respecto al espacio y al tiempo, no es aespacial, atemporal, sólo escapa al tiempo y al espacio concebidos como serie de acontecimientos en sí26.

La Forma es finalmente la nervadura común de la existencia y de la esencia, lo que vincula a los puntos espaciales y temporales a unos con otros sin transcender por ello el espacio y el tiempo, y de este modo hace posible a la vez las localizaciones puntuales y las unidades ideales (lo que Merleau-Ponty denomina "destello de mundo"). En realidad, el análisis de la Gestalt, llevado hasta el límite, nos hace salir de la lógica del tercero excluido, de la ontología parmenideana ${ }^{27}$. En efecto, decir que la forma no es la suma de sus partes sin ser otra cosa,

25 Nota inédita no publicada.

${ }^{26}$ VI, p. 258.

27 "La realidad de los organismos presupone un ser no parmenideano, una forma que escapa al dilema del ser y del no ser", Merleau-Ponty, M. (1995). La Nature. Notes de cours au Collège de France, Paris: Seuil, p. 239. 
equivale a decir que lo propio de lo percibido es de no ser sólo lo que él es ${ }^{28}$, que existe en el modo del exceso de sí mismo, "más que unidad y más que identidad", "sobresaturado", según las expresiones de Simondon. En otros términos, en cuanto que ella no es distinta de lo que ella estructura y al mismo tiempo no confundiéndose con aquello, la forma es un modo de unidad inmediata del ser y del no-ser, una diferencia pura o asignable. Se comprende por eso por qué Merleau-Ponty define la trascendencia (lo percibido) como "la identidad en la diferencia" y la nada como "la diferencia de los idénticos"29: esas formulaciones son la expresión directa del modo de ser de la Gestalt. Esto equivale a decir que, descrita rigurosamente, la forma nos conduce a un modo de pensar totalmente antidialéctico: pensar la forma es pensar un exceso sobre sí mismo sin desarrollo ni mediación, una negatividad que no es el reverso de una posición final; es poner en el centro de lo percibido una potencia que nada la puede actualizar y que es por así decirlo su propio acto. Somos conscientes con ello hasta qué punto la conceptualidad elaborada por Merleau-Ponty en sus obras anteriores se encontraba en contradicción con su propósito. Pensar la forma a partir de ella misma, no es contraponer una filosofía de inspiración trascendental al naturalismo de los psicólogos; es comprender la unidad de lo uno y de lo múltiple, de la significación y de la existencia más allá de un modo de pensar dialéctico, que empieza siempre por ratificar la dualidad.

Por supuesto, es necesario preguntarse por lo que ocurre, en una perspectiva semejante, con el sujeto para quién existe una forma, la "conciencia perceptiva". Merleau-Ponty señala, en Lo visible y lo invisible: "Tener conciencia = tener una figura sobre un fondo -no se puede ir más allá"30. Se le debe entender al pie de la letra. Esto no significa que toda conciencia perceptiva tiene como objeto percibido una estructura del tipo figura-fondo, sino que el acontecimiento de la conciencia consiste en la emergencia de una figura sobre un fondo, es decir en el nacimiento de una forma. Por ello Merleau-Ponty puede afirmar un poco más

28 "Decir que hay trascendencia, ser a distancia, es decir que el ser está hinchado de no-ser o de posible, que no es sólo lo que es" (VI, p. 234).

${ }^{29}$ VI, p. 279 y 316.

${ }^{30}$ VI, p. 245. 
adelante que el para-sí es un "carácter indiscutible, pero derivado: es la culminación de la distancia en la diferenciación Presencia para sí es presencia para mundo diferenciado"31: no hay otra realidad en la presencia para sí que la diferenciación constitutiva del fenómeno. En fin, como lo descubre Patočka prácticamente al mismo tiempo, hay una autonomía del aparecer y la experiencia perceptiva debe ser concebida como subordinada a la emergencia de lo percibido: lejos que la forma esté constituida por una conciencia, como Merleau-Ponty lo pensó por mucho tiempo, sólo hay experiencia perceptiva en virtud de la forma, de modo que es por así decirlo la conciencia quién es "constituida" por la forma. Quedaría entonces por determinar el para quién hay forma, es decir cuál es el sentido de ser del sujeto perceptivo, en cuanto que no constituye, sino que condiciona la emergencia de la forma. Es evidentemente del lado del cuerpo que hay que buscar, pero de un cuerpo que no es ni, claro está, fragmento de extensión, ni soporte de sensaciones localizadas. Merleau-Ponty observa que "la pregnancia es lo que, en lo visible, exige de mí una exacta puesta a punto, define su exactitud. Mi cuerpo obedece a la pregnancia, le 'responde', es lo que se suspende en ella, carne que responde a la carne ${ }^{\prime 32}$. En efecto, si la forma es lo que hemos dicho, su aprehensión no puede relevar ni de una pura receptividad, puesto que es precisamente una forma, ni de un acto intelectual, puesto que se encuentra hundida en los contenidos y no se da sino que en filigrana. La forma es objeto de una elaboración, es decir que exige de mi cuerpo un movimiento, algo así como un tanteo o una aproximación. Articulado hasta el final, el análisis del sujeto correlativo de la forma conduciría a colocar la motricidad en el centro de la percepción. Podríamos con ello incluso demostrar que el concepto ontológico de Carne descansa, a fin de cuentas, en la pregnancia como modo de existir común al organismo y a lo percibido ${ }^{33}$.

En las dos primeras obras, Merleau-Ponty ponía de relieve una convergencia entre la psicología de la forma en lo que tiene de más profundo y la fenomenología husserliana: la forma no podía sino remitir a una conciencia perceptiva y el paso por la psicología de la forma permitía así rectificar la reducción husserliana

\footnotetext{
31 VI, p. 245.

32 VI, p. 262.

${ }^{33}$ Cf. VI, p. 304.
} 
en un sentido no idealista. Ahora bien, varios de los textos que Merleau-Ponty dedica a la forma en Lo visible y lo invisible hacen referencia a Heidegger, e incluso se inscriben en un debate con él. En particular, la Gestalt es referida al Wesen en sentido verbal por el cual Heidegger intenta caracterizar lo que G. Kahn denomina el éster [l'ester], movimiento por el que el ser viene a la estancia. Por ejemplo, "la Gestaltung no es ser por definición, esencialización -es Wesen (verbal), operación de éster, aparición de un Etwas de proyección" ${ }^{\prime 34}$. Ahora bien, en mi opinión, una comparación de este tipo no procura tanto asimilar la Gestalt al Wesen como a hacer aparecer en la Gestalt el sentido verdadero del Wesen. ¿No podemos entonces hacer la hipótesis de que la determinación merleau-pontiana del Ser a partir de la forma permite rectificar la caracterización heideggeriana de la diferencia ontológica, que podríamos demostrar que es todavía demasiado abstracta y general? Construyendo una ontología indirecta, es decir comprendiendo el Ser a partir de lo percibido, Merleau-Ponty se daría los medios para definir el elemento mismo a partir del cual puede ser pensada la diferencia del Ser y del ente: la forma es en efecto la unidad misma de la retirada y de la aparición. Es así como, el estudio de la relación de Merleau-Ponty con la psicología de la forma vendría a confirmar la necesidad de situar su pensamiento, según las palabras de Ricœur, más allá de Husserl y Heidegger.

${ }^{34}$ VI, p. 260. 